\title{
Quality-Adjusted Life Years and Disability- Adjusted Life Years Are Better With Concurrent Chemoradiation Therapy Than Induction Chemotherapy Followed by Chemoradiation Therapy in Nasopharyngeal Carcinoma
}

Mary R. Nittala ${ }^{1}$, Madhava R. Kanakamedala ${ }^{2}$, Eswar Mundra ${ }^{1}$, William C. Woods III ${ }^{1}$, Maria L. Smith ${ }^{1}$, Robert D. Hamilton ${ }^{3}$, Gina D. Jefferson ${ }^{4}$, Lana Jackson ${ }^{4}$, Satya Packianathan ${ }^{1}$, Srinivasan Vijayakumar ${ }^{1}$

1. Radiation Oncology, University of Mississippi Medical Center, Jackson, USA 2. Radiation Oncology, Texas Oncology, Amarillo, USA 3. Hematology and Medical Oncology, University of Mississippi Medical Center, Jackson, USA 4. Otolaryngology - Head and Neck Surgery, University of Mississippi Medical Center, Jackson, USA

Corresponding author: Mary R. Nittala, mnittala@umc.edu

\section{Abstract}

\section{Introduction}

As traditional measures such as overall survival (OS) or disease-free survival (DFS) alone do not give a holistic view of the outcomes of a treatment paradigm, we determine to add the evidence of quality-adjusted life year (QALY) and disability-adjusted life year (DALY) to the outcomes of the nasopharyngeal carcinoma patients (NCP) treated with definitive chemoradiation therapy (chemoRT) with or without induction chemotherapy (induction chemo).

\section{Methods}

This is a retrospective analysis of $85 \mathrm{NCPs}$ treated at an academic state institution. The OS estimated by the Kaplan-Meier method and the multivariate Cox regression model determined the co-variables associated with the OS. The relationship between QALYs gained and DALYs saved were calculated from age of the disease onset, duration of the disease, quality of life (QoL) and disability weights.

\section{Results}

Of the 85 eligible NCPs of this cohort, the disease frequency distribution per the World Health Organization (WHO) classification was $41.2 \%$ for Type-I, $42.4 \%$ for Type-II, and $16.5 \%$ for Type-III. The median follow-up (24 months). The five-year OS of patients treated with concurrent chemoRT $v s$. induction chemo followed by concurrent chemoRT was 54.7 vs. $14.8 \%$ for WHO Type I, 60.1 vs. $58.3 \%$ for WHO Type II, and 83.3 vs. $50.0 \%$ for WHO Type III $(\mathrm{p}=0.029)$. The average DALYs saved with concurrent chemoRT were 12.2 years $v s .5$ years for induction chemo followed by concurrent chemoRT. The average QALYs gained with concurrent chemoRT

Review began 01/07/2021 Review ended 01/27/2021 Published 01/30/2021

\section{() Copyright 2021}

Nittala et al. This is an open access article distributed under the terms of the Creative Commons Attribution License CC-BY 4.0., which permits unrestricted use, distribution, and reproduction in any medium, provided the original author and source are credited. were 6.9 years vs. 3.1 years for induction chemo followed by concurrent chemoRT.

\section{Conclusion}

Patients treated with concurrent chemoRT had an increased QoL when compared to induction chemo followed by concurrent chemoRT. The average DALYs saved were higher in the patients treated with concurrent chemoRT than treated with induction chemo followed by concurrent chemoRT.

Categories: Radiation Oncology, Other, Epidemiology/Public Health

Keywords: quality-adjusted life year, disability-adjusted life year, nasopharyngeal carcinoma

\section{Introduction}

In the United States, nasopharyngeal carcinoma (NPC) is a rare neoplasm of the head and neck (HN) region and accounts for only $2 \%$ of all HN cancers; less than 1:10,000 persons are diagnosed with NPC each year and 129,000 new cases were diagnosed in 2018 [1,2]. Worldwide, however, it is the 18th and 22nd most commonly occurring cancer in men and women, respectively [3]. It is more common in Asian populations such as those originating from Southern China and Southeast Asia as well as in Eskimo and North African population, with annual incidence rates approaching 50 cases per 100,000 persons [4].

Nasopharyngeal carcinoma differs from other HN cancers in epidemiology, distant metastasis rates, and its association with the Epstein-Barr virus (EBV) [5]. The major source of morbidity and mortality in NPC patients comes from its high risk of distant metastasis. The World Health Organization (WHO) has classified NPC into three types based on histology: keratinizing squamous cell carcinoma (Type I), differentiated non- 
keratinizing carcinoma (Type II), and undifferentiated non-keratinizing carcinoma (Type III) [6,7]. In the US, about $25 \%$ of NPC patients have keratinizing carcinoma while $12 \%$ are differentiated non-keratinizing and $63 \%$ have the undifferentiated non-keratinizing histology [7].

Treatment for NPC depends upon the tumor stage and the patient's performance status [8]. Because of the operative morbidity associated with the anatomic location of these tumors, they are traditionally treated with radiation therapy (RT) [8,9]. Both non-keratinizing carcinomas (Type II \& III) respond better to radiation compared to the keratinizing carcinoma (Type I). The five-year overall survival rates for nonkeratinizing NPC is $51 \%$ vs. $6 \%$ for the keratinizing NPC [10]. Previous studies have suggested that OS rates of NPC patients were not significantly improved by induction chemotherapy (induction chemo) followed by concurrent chemoRT compared to concurrent chemoRT [11], and could even worsen the survival of stage II NPC patients [12].

A patient's pre-treatment health-related quality of life (HRQoL) has been shown to be a significant independent predictor of locoregional disease control in HN cancer patients [13]. Thus, an understanding of the contribution of various treatment modalities to a patient's QoL is very important as it because it helps to evaluate the type of treatment the $\mathrm{HN}$ cancer patients may need and also identify impairments in the ability to tolerate a specific treatment approach [14].

Traditionally, QoL is often evaluated using health survey questionnaires such as the Quality of Life Questionnaire C30 (QLQ-C30) or the European Organization for Research and Treatment of Cancer Quality of Life Questionnaire (EORTC QLQ) Head and Neck Cancer-Specific Module (H\&N35) [15]. When questionnaires are unavailable, QoL is often estimated by alternative methods of quality-adjusted life year (QALY) and disability-adjusted life year (DALY) [16].

QALY captures both the impact of a treatment on a patient's length of life and also its impact on their HRQoL [17]. DALY is a summary measure of public health widely used to quantify the burden of disease [18]. In this study, we used QALYs and DALYs to assess the impact of QoL on survival outcomes of NPC patients treated with concurrent chemoRT with or without induction chemotherapy.

This article was previously presented as a meeting abstract at the 2019 AACR-AHNS Head and Neck Cancer Conference at Austin, TX, USA on April 29, 2019. This article was previously posted to the Research Square preprint server on August 26, 2020.

\section{Materials And Methods}

\section{Patients}

The study subjects were 96 NPC patients diagnosed and treated between 1994 and 2018 at the University of Mississippi Medical Center (UMMC), Jackson, MS, USA. Institutional review board approval was obtained for the retrospective analysis. A browser-based database -- Research Electronic Data Capture (REDCap; Vanderbilt University, Nashville, TN, USA) -- was used to gather and store the patient information. Written consent was waived secondary to the retrospective nature of the study and patient identifiers were removed before the data were extracted for analysis. Nine patients with an unknown WHO class of tumor were excluded from the study and hence, 85 patients were included in the final analyses.

\section{Treatment methods}

Fifty-one patients (60\%) received concurrent chemoRT consisting of weekly cisplatin $(40 \mathrm{mg} / \mathrm{m} 2)$ during radiotherapy for a maximum of seven cycles, beginning on the first day of radiotherapy. Twenty patients (23.5\%) received induction chemo with docetaxel, cisplatin, and fluorouracil (TPF) followed by concurrent chemoRT as described above, while 14 patients (16.5\%) received no treatment.

\section{QoL assessments}

QALY: Is a measure of the value of health outcome and combines length and QoL into a single unit. It is calculated simply by multiplying the duration of time spent in a health stage by the HRQoL weighting associated with that health state.

DALY: Is a common measurement unit for morbidity and mortality. It is composed of years lived with the disability (YLD) and years of life lost (YLL) due to premature mortality associated with that disability.

$\mathrm{DALY}=\mathrm{YLD}+\mathrm{YLL}$

$\mathrm{YLD}=$ number of cases $\mathrm{x}$ duration till remission (or) death $\mathrm{x}$ disability weight (DW)

YLL = number of deaths $\mathrm{x}$ life expectancy $(\mathrm{LE})$ at the age of death 
DW is a scale from zero (perfect health) to one (worst possible health state) and LE was obtained from the actuarial life tables.

\section{Statistical analysis}

The overall survival (OS) was defined by the number of days from the date of initial diagnosis until the date of death or the last contact. The censored cases included patients without death at the time of the last follow up. The Kaplan-Meier method was used to estimate the OS rates and the univariate significance of differences among survival curves was calculated by the log-rank test. The co-variables associated with the OS were determined by the multivariate Cox regression model. Hazards ratio (HR) was used to estimate the time-to-event outcome with associated $95 \%$ confidence intervals (CIs) and p-values $\leqslant 0.05$ were considered statistically significant. Average QALYs gained and average DALYs saved were calculated for all the variables stratified by the tumor histology. The SPSS 24.0 software (IBM Corp., Armonk, NY, USA) was used for data analyses.

\section{Results}

\section{Patient characteristics}

In this 85 NPC cohort, there were 55 (64.7\%) male patients and 30 (35.3\%) female patients. The median age was 56 years (range, 19-86 years). Out of the 85 NPC patients, 35 (41.2\%) had WHO Type I disease, 36 (42.4\%) had WHO Type II disease, and 14 (16.5\%) had WHO Type III disease. Fifty-seven (67.1\%) were insured while 28 (32.9\%) had no insurance. More than half of the patients 60 (70.6\%) had a history of smoking and the use of alcohol was 50-50 in this group. The stage distribution in this cohort was Stage I (5.9\%), Stage II (9.4\%), Stage III (16.5\%), Stage IV (52.9\%), and Unknown Stage (15.3\%). Fifty-one (60\%) were treated with concurrent chemoRT, 20 (23.5\%) with induction chemo followed by the chemoRT, and 14 (16.5\%) NPC patients were hospice (Table 1).

\begin{tabular}{|c|c|c|c|c|c|}
\hline \multirow{2}{*}{ Variable } & WHO Type I & WHO Type II & WHO Type III & All patients & $p$-value \\
\hline & $\mathrm{n}=35(41.2 \%)$ & $\mathrm{n}=36(42.4 \%)$ & $\mathrm{n}=14(16.5 \%)$ & $\mathrm{n}=85(100 \%)$ & \\
\hline \multicolumn{6}{|l|}{ GENDER/SEX } \\
\hline Male & 24 (68.6\%) & 20 (55.6\%) & 11 (78.6\%) & 55 (64.7\%) & \\
\hline Female & $11(31.4 \%)$ & 16 (44.4\%) & $3(21.4 \%)$ & 30 (35.3\%) & 0.256 \\
\hline \multicolumn{6}{|l|}{ AGE } \\
\hline$<35$ years & $1(2.9 \%)$ & 5 (13.9\%) & $3(21.4 \%)$ & 9 (10.6\%) & \\
\hline $35-60$ years & 22 (62.9\%) & 16 (44.4\%) & 6 (42.9\%) & 44 (51.8\%) & \\
\hline$>60$ years & $12(34.3 \%)$ & $15(41.7 \%)$ & $5(35.7 \%)$ & 32 (37.6\%) & 0.232 \\
\hline \multicolumn{6}{|l|}{ ETHNICITY } \\
\hline Black & 16 (45.7\%) & 22 (61.1\%) & 9 (64.3\%) & 47 (55.3\%) & \\
\hline White & 17 (48.6\%) & 12 (33.3\%) & $4(28.6 \%$ & 33 (38.8\%) & \\
\hline Others & $2(5.7 \%)$ & $2(5.6 \%)$ & $1(7.1 \%)$ & $5(5.9 \%)$ & 0.633 \\
\hline \multicolumn{6}{|l|}{ INSURANCE } \\
\hline Medicaid & $9(25.7 \%)$ & 11 (30.6\%) & $3(21.4 \%)$ & $23(27.1 \%)$ & \\
\hline Medicare & $8(22.9 \%)$ & 7 (19.4\%) & $4(28.6 \%)$ & 19 (22.4\%) & \\
\hline Private & 5 (14.3\%) & $8(22.2 \%)$ & 2 (14.3\%) & 15 (17.6\%) & \\
\hline Self-pay & $13(37.1 \%)$ & 10 (27.8\%) & 5 (35.7\%) & 28 (32.9\%) & 0.921 \\
\hline \multicolumn{6}{|l|}{ DISIANCE } \\
\hline$<30$ miles & $12(34.3 \%)$ & $7(19.4 \%)$ & $5(35.7 \%)$ & $24(28.2 \%)$ & \\
\hline 30 - 75 miles & 6 (17.1\%) & $8(22.2 \%)$ & $1(7.1 \%)$ & $15(17.6 \%)$ & \\
\hline$>75$ miles & 17 (48.6\%) & 21 (58.3\%) & $8(57.1 \%)$ & 46 (54.1\%) & 0.499 \\
\hline
\end{tabular}




\section{Cureus}

\begin{tabular}{|c|c|c|c|c|c|}
\hline Smoker & 29 (82.9\%) & 22 (61.1\%) & 9 (64.3\%) & $60(70.6 \%)$ & \\
\hline Non-Smoker & $6(17.1 \%)$ & $14(38.9 \%)$ & $5(35.7 \%)$ & 25 (29.4\%) & 0.113 \\
\hline \multicolumn{6}{|l|}{ ALCOHOL } \\
\hline Drinker & $22(62.9 \%)$ & $14(38.9 \%)$ & 7 (50.0\%) & $43(50.6 \%)$ & \\
\hline Non-Drinker & 13 (37.1\%) & 22 (61.1\%) & $7(50.0 \%)$ & 42 (49.4\%) & 0.130 \\
\hline \multicolumn{6}{|l|}{ TUMOR STAGE } \\
\hline Stage I & $2(5.7 \%)$ & $1(2.8 \%)$ & $2(14.3 \%)$ & $5(5.9 \%)$ & \\
\hline Stage II & $3(8.6 \%)$ & $5(13.9 \%)$ & $0(0.0 \%)$ & $8(9.4 \%)$ & \\
\hline Stage III & $6(17.1 \%)$ & $8(22.2 \%)$ & $0(0.0 \%)$ & $14(16.5 \%)$ & \\
\hline Stage IV & $21(60.0 \%)$ & $19(52.8 \%)$ & $5(35.7 \%)$ & 45 (52.9\%) & \\
\hline Unknown Stage & $3(8.6 \%)$ & $3(8.3 \%)$ & $7(50.0 \%)$ & $13(15.3 \%)$ & 0.005 \\
\hline \multicolumn{6}{|l|}{ TREATMENT } \\
\hline Concurrent chemoRT & $21(60.0 \%)$ & $24(66.7 \%)$ & $6(42.9 \%)$ & $51(60.0 \%)$ & \\
\hline Induction chemo + chemoRT & $9(25.7 \%)$ & $8(22.2 \%)$ & $3(21.4 \%)$ & $20(23.5 \%)$ & \\
\hline Hospice & 5 (14.3\%) & $4(11.1 \%)$ & $5(35.7 \%)$ & 14 (16.5\%) & 0.292 \\
\hline
\end{tabular}

TABLE 1: Demographic and clinical description of the nasopharyngeal study population by histology - WHO classification

WHO = World Health Organization; induction chemo = induction chemotherapy; chemoRT = chemoradiation therapy

\section{QoL data}

The average QALYs gained and average DALYs saved for different variables was stratified by the histology of the NPC in the study population (Table 2). The average LE for this 85-patient NPC cohort was 34.51 years; average DALYs saved with treatment were 20.06 years and average QALYs gained with treatment were 11.77 years. The DALYs saved with concurrent chemoRT were 12.2 years $v s$. five years with induction chemo followed by concurrent chemoRT. The QALYs gained with concurrent chemoRT were 6.9 years vs. 3.1 years with induction chemo followed by concurrent chemoRT.

\begin{tabular}{|l|l|l|}
\hline Variable & Avg. QALYs Gained & Avg. DALYs Saved \\
\hline GENDER/SEX & 11.63 & 20.19 \\
Male & 10.06 & 18.53 \\
Female & & \\
AGE & 17.01 & 26.01 \\
$<35$ years & 12.35 & 21.37 \\
35 - 60 years & 7.67 & 15.38 \\
$>60$ years & & \\
ETHNICITY & 11.53 & 20.09 \\
Black & 11.51 & 19.62 \\
White & 4.06 & 15.00 \\
Others & & 22.75 \\
INSURANCE & 13.55 & \\
Medicaid & & \\
\hline
\end{tabular}




\section{Cureus}

\begin{tabular}{|c|c|c|}
\hline Medicare & 8.67 & 15.77 \\
\hline Private & 8.33 & 16.63 \\
\hline Self-pay & 12.17 & 21.22 \\
\hline \multicolumn{3}{|l|}{ DISTANCE } \\
\hline$<30$ miles & 11.49 & 20.73 \\
\hline $30-75$ miles & 13.10 & 20.35 \\
\hline$>75$ miles & 10.21 & 18.78 \\
\hline \multicolumn{3}{|l|}{ SMOKING } \\
\hline Smoker & 10.86 & 19.43 \\
\hline Non-Smoker & 11.62 & 20.04 \\
\hline \multicolumn{3}{|l|}{ ALCOHOL } \\
\hline Drinker & 11.03 & 19.84 \\
\hline Non-Drinker & 11.13 & 19.36 \\
\hline \multicolumn{3}{|l|}{ TUMOR STAGE } \\
\hline Stage I & 7.71 & 15.67 \\
\hline Stage II & 8.07 & 17.28 \\
\hline Stage III & 11.20 & 18.52 \\
\hline Stage IV & 12.40 & 21.27 \\
\hline Unknown Stage & 9.49 & 17.95 \\
\hline \multicolumn{3}{|l|}{ TREATMENT } \\
\hline Concurrent ChemoRT & 10.84 & 19.90 \\
\hline Induction Chemo + ChemoRT & 12.61 & 20.22 \\
\hline Hospice & 9.75 & 1 \\
\hline
\end{tabular}

\section{TABLE 2: QALYs and DALYs of the nasopharyngeal study population}

QALY = quality-adjusted life year; DALY = disability-adjusted life year; chemoRT = chemoradiation therapy; Induction chemo = induction chemotherapy

\section{Survival outcomes}

The median follow up for this study population was 24 months. The five-year OS of WHO Type I was $34.8 \%$, Type II 58\%, and Type III 77.1\% ( $\mathrm{p}=0.042$ ). The median survival of WHO Type I was 34 months, Type II 194 months, and Type III 123 months (Figure 1). The OS curve for different tumor stages are represented in Figure 1. For all histologies, the five-year OS for Stage I was $80.0 \%$, Stage II $57.1 \%$, Stage III $52.6 \%$, Stage IV 44.5\%, and Stage Unknown 64.5\% ( $\mathrm{p}=0.321)$. 


\section{Cureus}

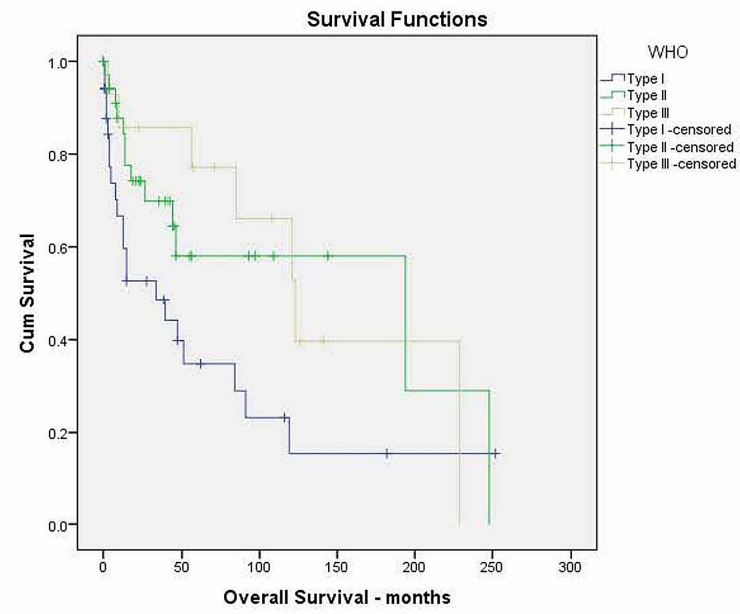

Number of NCP at Risk categorized by WHO Types
\begin{tabular}{|l|r|r|r|r|r|r|r|}
\hline Type I & 34 & 7 & 4 & 2 & 1 & 0 & 0 \\
\hline Type II & 35 & 8 & 4 & 3 & 2 & 1 & 0 \\
\hline Type III & 13 & 10 & 6 & 1 & 0 & 0 & 0 \\
\hline
\end{tabular}

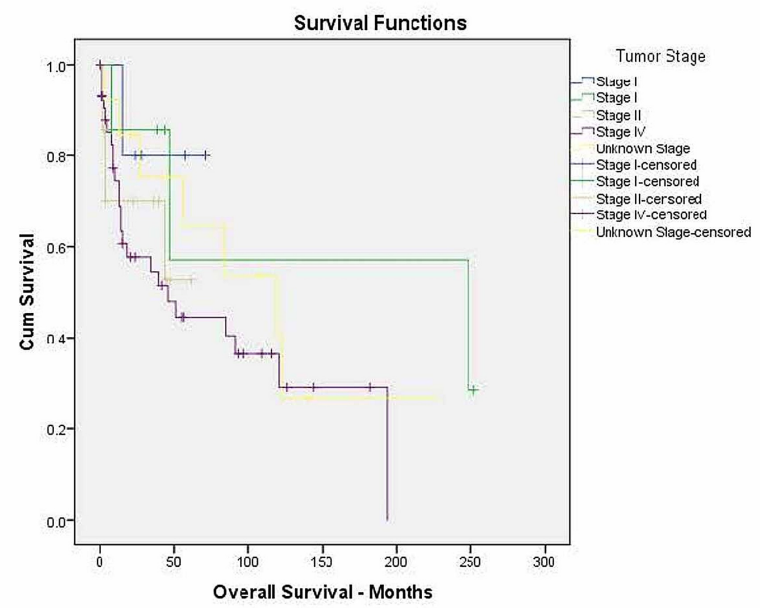

Number of NCP at Risk categorized by Tumor Stage Types
\begin{tabular}{|l|r|r|r|r|r|r|r|}
\hline Stage I & 4 & 2 & 0 & 0 & 0 & 0 & 0 \\
\hline Stage II & 7 & 2 & 1 & 1 & 1 & 0 & 0 \\
\hline Stage III & 13 & 1 & 0 & 0 & 0 & 0 & 0 \\
\hline Stage IV & 44 & 13 & 7 & 2 & 0 & 0 & 0 \\
\hline $\begin{array}{l}\text { Stage } \\
\text { Unknown }\end{array}$ & 12 & 7 & 4 & 1 & 0 & 0 & 0 \\
\hline
\end{tabular}

FIGURE 1: Kaplan-Meier overall survival curves for the study population by tumor histology (WHO types) and tumor stages

WHO = World Health Organization; NCP = nasopharyngeal carcinoma patients

\section{Univariate analyses}

In the univariate analyses, the variables of gender, age, ethnicity, insurance, distance traveled to the treatment facility, alcohol history, and the treatment modality were significantly associated with the OS (Table 3). The OS curves for the treatment modalities used are represented in Figure 2. Five-year OS of NCPs treated by concurrent chemoRT for WHO Type I was 54.7\%, WHO Type II 60.1\%, and WHO Type III $83.3 \%$ $(p=0.029)$. Five-year OS of NCPs treated by induction chemo followed by concurrent chemoRT for WHO Type I was $14.8 \%$, WHO Type II 58.3\%, and WHO Type III 50.0\% ( $\mathrm{p}=0.029)$. 


\section{Cureus}

Variable

Gender/sex

Age

Ethnicity

Insurance

Distance

Smoking

Alcohol

Treatment log rank p-value

0.013

0.013

0.042

0.026

0.041

0.960

0.010

0.029

TABLE 3: Univariate analysis by tumor histology 


\section{Cureus}

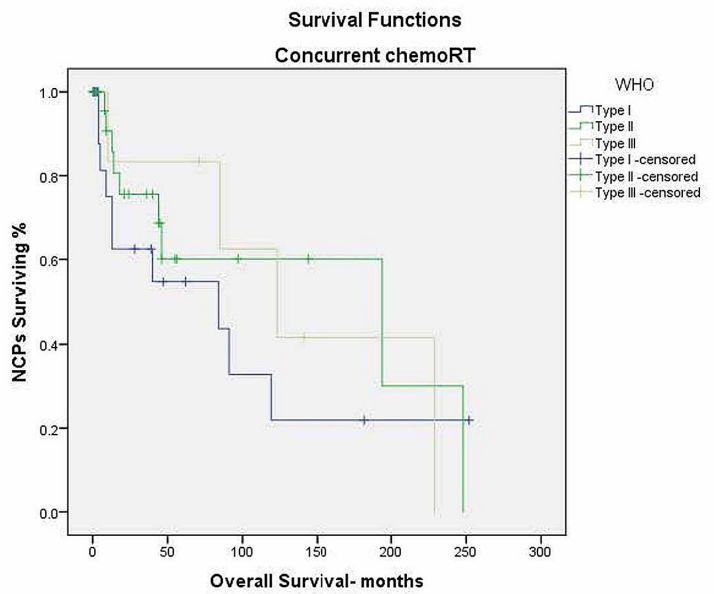

Number of NCP treated concurrent chemoRT at Risk categorized by WHO Types

\begin{tabular}{|l|l|l|l|l|l|l|l|}
\hline Type I & 8 & 2 & 0 & 0 & 0 & 0 & 0 \\
\hline Type II & 7 & 2 & 0 & 0 & 0 & 0 & 0 \\
\hline Type III & 2 & 1 & 0 & 0 & 0 & 0 & 0 \\
\hline
\end{tabular}

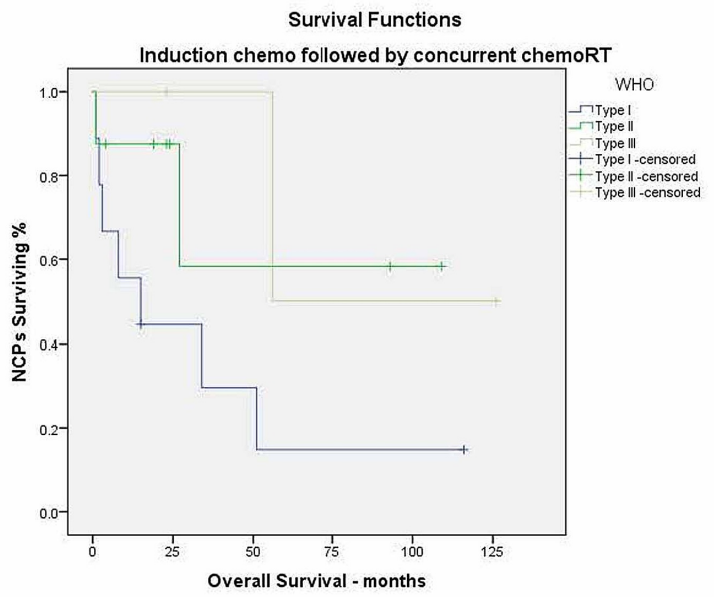

Number of NCP treated by induction chemo followed by concurrent chemoRT at Risk categorized by WHO Types

\begin{tabular}{|l|r|r|r|r|r|r|}
\hline Type I & 20 & 10 & 6 & 5 & 3 & 2 \\
\hline Type II & 23 & 13 & 6 & 4 & 3 & 2 \\
\hline Type III & 5 & 4 & 4 & 3 & 3 & 2 \\
\hline
\end{tabular}

FIGURE 2: The Kaplan-Meier overall survival curves for NCPs treated with concurrent chemoRT vs. NCPs treated with induction chemo followed by concurrent chemoRT

WHO = World Health Organization; NCP = nasopharyngeal carcinoma patients; chemoRT = chemoradiation therapy; induction chemo $=$ induction chemotherapy

\section{Multivariate analyses}

The variables which were significantly associated with survival outcomes were included in the Cox proportional hazards regression model (Table 4). In the multivariate analyses, only tumor histology was significantly associated with OS, with HR of 0.61 (95\% CI 0.39-0.94), $\mathrm{p}=0.027$. 


\section{Cureus}

\begin{tabular}{|c|c|c|}
\hline Variable & HR (95\% Cl) & $p$-value \\
\hline Gender/sex & $0.52(0.24-1.15)$ & 0.111 \\
\hline Age & $0.84(0.48-1.47)$ & 0.557 \\
\hline Ethnicity & $0.87(0.50-1.53)$ & 0.879 \\
\hline Insurance & $1.13(0.85-1.49)$ & 0.389 \\
\hline Distance & $0.87(0.58-1.29)$ & 0.496 \\
\hline Alcohol & $1.40(0.71-2.78)$ & 0.324 \\
\hline Treatment & $0.88(0.48-1.59)$ & 0.682 \\
\hline Tumor histology & $0.61(0.39-0.94)$ & 0.027 \\
\hline
\end{tabular}

TABLE 4: Multivariate Cox regression analysis

$\mathrm{HR}=$ hazard ratio; $\mathrm{Cl}=$ confidence interval

\section{Discussion}

Nasopharyngeal carcinoma is a rare neoplasm of the HN in the United States and accounts for only $2 \%$ of all the HN cancers $[1,2]$. Our data for this cohort of patients, accounting for $2.4 \%$ of all the patients in our HN cancer database, is consistent with the literature. Patients with NPC are mostly males with a known history of tobacco and heavy alcohol use [19], and our data reflects what has been previously reported as $65 \%$ were males with $71 \%$ had a known history of tobacco and $51 \%$ had a history of heavy alcohol use. Nasopharyngeal carcinoma is more common in Asians/Pacific Islanders, who are diagnosed six times more frequently than Caucasian and Hispanic people [20], and in whom the disease can occur at any age, including children, while about $50 \%$ of people with the disease are 55 years or younger. Our patient cohort consisted of $39 \%$ Caucasian and $55 \%$ African Americans, which is most likely due to our regional demographics, while $53 \%$ of the cohort were younger than 60 years.

Generally, the NPC histology distribution in the United States is keratinizing carcinoma (Type I) $25 \%$, differentiated non-keratinizing (Type II) 12\%, and undifferentiated non-keratinizing (Type III) 63\%. In our cohort, these histologies, however, were represented by $41 \%, 42 \%$, and $17 \%$, respectively. It is possible that these differences are due to the ethnic demographics of our patient population, with very few Asian/Pacific Islanders represented. In the literature, the reported five-year OS for non-keratinizing NPC is $51 \%$ vs. $6 \%$ for keratinizing NPC [10]. In our cohort though, the five-year OS was $66 \%$ vs. 38\%, with a median survival of 24 months.

It is established that NPC patients have a higher likelihood of living cancer-free for an extended period of time with treatment methods that utilize radiation therapy (RT) alone or in combination with chemotherapy [21]. Many reports have suggested that treatments delivered with more advanced RT techniques show a significant trend toward improving QoL outcomes $[22,23]$. In a prospective randomized trial comparing the QoL of NPC patients treated with two-dimensional radiation therapy (2DRT) vs. intensity-modulated radiotherapy (IMRT), it was shown that IMRT significantly improved salivary flow after RT, although no other scale being measured significantly improved, except physical and role functioning, as assessed by the EORTC QLQ-C30 [24]. Some studies have noted that the use of three-dimensional chemoRT/IMRT reduced the HN-related symptom scales to some extent and thereby improved QoL [25]. Besides the RT technique used, socioeconomic status, comorbidity, and tumor site were also found to be significant prognostic predictors of a patient's HR-QoL outcome. Patients who had higher economic status, higher education levels, employed status, and fewer comorbidities tended to have better HR-QoL [26]. Some studies have reported that female gender, higher cancer stage, and combination treatment were associated with more symptoms and worse HR-QoL [27, 28]. Few studies had explored the prognostic significance of QoL in QLQC30 questionnaires for NPC patients. Studies conducted to evaluate the impact of replanning on the QoL during IMRT showed that replanning had both statistically and clinically significant improvements in QoL scales [29]. A study by Tan et.al. [30] indicates that induction chemo followed by chemoRT was not shown to improve OS compared to definitive chemoRT. In our study, we found that the OS of concurrent chemoRT vs. induction chemo followed by concurrent chemoRT was $32.8 \%$ vs. $14.8 \%(\mathrm{p}=0.029)$.

Many studies have previously used the EORTC health survey questionnaires to investigate the QoL of NPC patients. For our analysis, however, we were unable to estimate patient QoL using the questionnaire method as our institution started using health survey questionnaires only recently. Thus, in order to calculate QoL without available survey data, we used QALYs and DALYs as health outcome measures that account for both 
longevity and QoL [16]. In our investigation, we found that the average LE for our 85 NPC cohort was 34.56 years, the average DALYs saved with treatment were 20.06 years, and the average QALYs gained with treatment were 11.77 years. The DALYs saved with concurrent chemoRT were 12.2 years $v s$. five years with induction chemo followed by concurrent chemoRT. The QALYs gained using concurrent chemoRT were 6.9 years vs. 3.1 years with induction chemo followed by concurrent chemoRT.

As with all retrospective analyses, our study is not without its limitations. As we had no HR-QoL data available, potential selection bias is one of the limitations. The QALY approach used in this study does not explicitly incorporate equity weights, which can be a challenge when comparing treatment interventions. In order to overcome the challenges posed by using the QALY approach, we utilized an alternative method, DALY, to compare the impact of disease burden among treatment modalities.

\section{Conclusions}

Our retrospective analysis reports OS findings in NPC similar to that in previous studies, indicating that induction chemo followed by chemoRT was not shown to improve OS compared to definitive chemoRT. In addition, our data suggest that patients treated with concurrent chemoRT had an improved QoL compared to that of patients treated with induction chemo followed by concurrent chemoRT. The average DALYs saved and average QALYs gained were higher in the patients treated with concurrent chemoRT. However, there may be some yet unidentified factors or some specific functional domains related to the QoL which were unappreciated in this analysis.

\section{Additional Information \\ Disclosures}

Human subjects: Consent was obtained or waived by all participants in this study. University of Mississippi Medical Center Institutional Review Board issued approval 2010-0252. Your Continuing Review was reviewed and approved by the Expedited Review Process on06/29/2020. You may continue this research. Animal subjects: All authors have confirmed that this study did not involve animal subjects or tissue. Conflicts of interest: In compliance with the ICMJE uniform disclosure form, all authors declare the following: Payment/services info: All authors have declared that no financial support was received from any organization for the submitted work. Financial relationships: All authors have declared that they have no financial relationships at present or within the previous three years with any organizations that might have an interest in the submitted work. Other relationships: All authors have declared that there are no other relationships or activities that could appear to have influenced the submitted work.

\section{References}

1. Key statistics for nasopharyngeal cancer. (2019). Accessed: April 10, 2020: https://www.cancer.org/cancer/nasopharyngeal-cancer/about/key-statistics.html.

2. Qu S-HI, Zell A, Ziogas A, Anton-Culver H: Epidemiology of nasopharyngeal carcinoma in the United States: improved survival of Chinese patients within the keratinizing squamous cell carcinoma histology. Ann Oncol. 2007, 18:29-35. 10.1093/annonc/mdl320

3. Global Burden of Disease Cancer Collaboration: Global, regional, and national cancer incidence, mortality, years of life lost, years lived with disability, and disability-adjusted life-years for 32 cancer groups, 1990 to 2015: a systematic analysis for the Global Burden of Disease Study. JAMA Oncol. 2017, 3:524-548. 10.1001/jamaoncol.2016.5688

4. Wee JTS, Ha TC, Loong SL, Qian CN: Is nasopharyngeal cancer really a “Cantonese cancer”? . Chin J Cancer. 2010, 29:517-526. 10.5732/cjc.009.10329

5. Raab-Traub N: Epstein-Barr virus in the pathogenesis of NPC . Semin Cancer Biol. 2002, 12:431-441. 10.1016/S1044579X0200086X

6. Stelow EB, Wenig BM: Update from the 4th edition of the World Health Organization Classification of Head and Neck Tumours: nasopharynx. Head and Neck Pathol. 2017, 11:16-22. 10.1007/s12105-017-0787-0

7. Thompson LD: Update on nasopharyngeal carcinoma. Head Neck Pathol. 2007, 1:81-88. 10.1007/s12105007-0012-7

8. Wang Y, Zhang Y, Ma S: Racial differences in nasopharyngeal carcinoma in the United States . Cancer Epidemiol. 2013, 37:793-802. 10.1016/j.canep.2013.08.008

9. Fang FM, Tsai WL, Go SF, et al.: Implications of quantitative tumor and nodal regression rates for nasopharyngeal carcinomas after 45 Gy of radiotherapy. Int J Radiat Oncol. 2001, 50:961-969. 10.1016/S0360-3016(01)01531-0

10. Reddy SP, Raslan WF, Gooneratne S, Kathuria S, Marks JE: Prognostic significance of keratinization in nasopharyngeal carcinoma. Am J Otolaryngol. 1995, 16:103-108. 10.1016/0196-0709(95)90040-3

11. Chen L, Hu CS, Chen XZ, et al.: Concurrent chemoradiotherapy plus adjuvant chemotherapy versus concurrent chemoradiotherapy alone in patients with locoregionally advanced nasopharyngeal carcinoma: a phase 3 multicenter randomized controlled trial. Lancet Oncol. 2012, 13:163-171. 10.1016/S14702045(11)70320-5

12. Jin T, Zhang Q, Luo DH, et al.: Concurrent chemoradiotherapy with or without induction chemotherapy for patients with sthae II nasopharyngeal carcinoma: an update. Trans Oncol. 2020, 13:25-31. 10.1016/j.tranon.2019.08.007

13. Siddiqui F, Pajak TF, Watkins-Bruner D, et al.: Pretreatment quality of life predicts for locoregional control in head and neck cancer patients: a radiation therapy oncology group analysis. Int J Radiat Oncol Biol Phys. 
2008, 70:353-360. 10.1016/j.ijrobp.2007.06.024

14. Karvonen-Gutierrez CA, Ronis DL, Fowler KE, Terrell JE, Gruber SB, Duffy SA: Quality of life scores predict survival among patients with head and neck cancer. J Clin Oncol. 2008, 26:2754-2760.

10.1200/JCO.2007.12.9510

15. Arraras JI, Kuljanic-Vlasic K, Bjordal K, et al.: EORTC QLQ-INFO26: a questionnaire to assess information given to cancer patients a preliminary analysis in eight countries. Psychooncology. 2007, 16:249-254. 10.1002/pon.1047

16. Sanders GD, Neumann PJ, Basu A, et al.: Recommendations for conduct, methodological practices, and reporting of cost-effectiveness analyses: second panel on cost-effectiveness in health and medicine. JAMA. 2016, 316:1093-1103. 10.1001/jama.2016.12195

17. Whitehead SJ, Ali S: Health outcomes in economic evaluation: the QALY and utilities . Br Med Bull. 2010, 96:5-21. 10.1093/bmb/ldq033

18. Devleesschauwer B, Havelaar AH, de Noordhout CM, et al.: Calculating disability-adjusted life years to quantify burden of disease. Int J Public Health. 2014, 59:565-569. 10.1007/s00038-014-0552-Z

19. Voltzke KJ, Lee YCA, Zhang ZF, et al.: Racial differences in the relationship between tobacco, alcohol, and the risk of head and neck cancer: pooled analysis of US studies in the INHANCE Consortium. Cancer Causes Control. 2018, 29:619-630. 10.1007/s10552-018-1026-z

20. Hashibe M: Risk factors: tobacco and alcohol. Epidemiology, Pathogenesis, and Prevention of Head and Neck Cancer. Olshan AF (ed): Springer, New York; 2010. 65-85. 10.1007/978-1-4419-1472-9

21. Budach W, Bolke E, Kammers K, Gerber PA, Orth K, Gripp S, Matuschek C: Induction chemotherapy followed by concurrent radio-chemotherapy versus concurrent radio-chemotherapy alone as treatment of locally advanced squamous cell carcinoma of the head and neck (HNSCC): a meta-analysis of randomized trials. Radiother Oncol. 2016, 118:238-243. 10.1016/j.radonc.2015.10.014

22. Chen AM, Daly ME, Farwell DG, Vazquez, E Courquin J, Lau DH , Purdy JA: Quality of life among long-term survivors of head and neck cancer treated by intensity-modulated radiotherapy. JAMA Otolaryngol Head Neck Surg. 2014, 140:129-133. 10.1001/jamaoto.2013.5988

23. Zhang B, Mo Z, Du W, Wang Y, Liu L, Wei Y: Intensity-modulated radiation therapy versus 2D-RT or 3DCRT for the treatment of nasopharyngeal carcinoma: a systematic review and meta-analysis. Oral Oncol. 2015, 51:1041-1046. 10.1016/j.oraloncology.2015.08.005

24. Pow EHN, Kwong DLW, McMillan AS, Wong MCM, Sham IST, Leun LST, Leung WK: Xerostomia and quality of life after intensity-modulated radiotherapy vs. conventional radiotherapy for early-stage nasopharyngeal carcinoma: initial report on a randomized controlled clinical trial. Int J Radiat Oncol Biol Phys. 2006, 66:981991. 10.1016/j.ijrobp.2006.06.013

25. Lee AW, Ng WT, Chan LL, et al.: Evolution of treatment for nasopharyngeal cancer-success and setback in the intensity-modulated radiotherapy era. Radiother Oncol. 2014, 110:377-384. 10.1016/j.radonc.2014.02.003

26. Adler NE, Boyce WT, Chesney MA, Folkman S, Syme SL: Socioeconomic inequalities in health: no easy solution. JAMA. 1993, 269:3140-3145. 10.1001/jama.1993.03500240084031

27. Stanford-Moore G, Bradshaw PT, Weissler MC, et al.: Interaction between known risk factors for head and neck cancer and socioeconomic status: the Carolina Head and Neck Cancer Study. Cancer Causes Control. 2018, 29:863-873. 10.1007/s10552-018-1062-8

28. Gyllensten H, Koinberg I, Carlström E, Olsson LE, Olofsson EH: Economic evaluation of a person-centred care intervention in head and neck oncology: results from a randomized controlled trail. Support Care Cancer. 2019, 27:1825-1834. 10.1007/s00520-018-4436-2

29. Fang FM, Herng-Chia C, Wen-Rei K, et al.: Health-related quality of life for nasopharyngeal carcinoma patients with cancer-free survival after treatment. Int J Radiat Oncol Biol Phys. 2002, 53:959-968. 10.1016/S0360-3016(02)02838-9

30. Tan T, Lim WT, Fong KW, et al.: Concurrent chemo-radiation with or without induction gemcitabine, carboplatin, and paclitaxel: a randomized, phase $2 / 3$ trial in locally advanced nasopharyngeal carcinoma. Int J Radiat Oncol Biol Phys. 2015, 91:952-960. 10.1016/j.ijrobp.2015.01.002 